\title{
Neutrino oscillation effect on the indirect signal of neutralino dark matter from the Earth core
}

\author{
Nicolao Fornengo \\ Instituto de Física Corpuscular - C.S.I.C. \\ Departamento de Física Teòrica, Universitat de València \\ c./ Dr. Moliner 50, E-46100_Burjassot, València, Spain \\ E-mail: 'fornengo@flamenco.ific.uv.es'
}

ABstract: We investigate the effect induced by neutrino oscillation on the dark matter indirect detection signal which consists in a muon neutrino flux produced by neutralino annihilation in the Earth core. We consider the neutrino oscillation parameters relevant to the atmosferic neutrino deficit, both in the $\nu_{\mu} \rightarrow \nu_{\tau}$ and $\nu_{\mu} \rightarrow \nu_{s}$ cases.

\section{Introduction}

Many different techniques have been proposed for the detection of dark matter particles which could make up the halo of our Galaxy. Among the different possibilities, the detection of a neutrino flux by means of neutrino telescopes represents certainly an interesting tool, which is already at the level of imposing some (mild) constraint on the particle physics properties of the neutralino, the most interesting and studied dark matter candidate ${ }_{i=1}^{i}$. This particle is present in all the supersymmetric extensions of the standard model as a linear combination of the superpartners of the neutral gauge and higgs fields. In the present paper we will perform our calculations in the minimal supersymmetric extension of the standard model (MSSM), for a definition of which we refer to Ref. [2in] and to the references therein quoted.

\section{Up-going muons from neutralino annihilation in the Earth}

The neutrino flux has origin from neutralino pair annihilation inside the Earth where these dark matter particles can be accumulated after having been captured by gravitational trapping. The differential flux is

$$
\Phi_{(-)}^{0}\left(E_{\nu}\right) \equiv \frac{d N_{(-)}}{d E_{\nu}}=\frac{\Gamma_{A}}{4 \pi d^{2}} \sum_{F, f} B_{\chi f}^{(F)} \frac{d N_{f(-)}^{(-)}}{d E_{\nu}}
$$

where $\Gamma_{A}$ denotes the annihilation rate, $d$ is the distance of the detector from the source (i.e. the center of the Earth), $F$ lists the neutralino pair annihilation final states, $B_{\chi f}^{(F)}$ denotes the branching ratios into heavy quarks, $\tau$ lepton and gluons in the channel $F$. The spectra $d N_{f \nu_{\mu}^{(-)}} / d E_{\nu}$ are the differential distributions of the (anti) neutrinos generated by the $\tau$ and by hadronization of quarks and gluons and the subsequent semileptonic decays of the produced hadrons. For de-

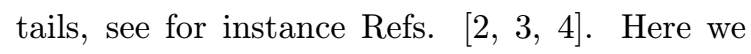
only recall that the annihilation rate depends, through its relation with the capture rate of neutralinos in the Earth, on some astrophysical parameters, the most relevant of which is the local density $\rho_{l}$.

The neutrino flux is produced in the inner part of the Earth [2] and propagates toward a detector where it can be detected as a flux of up-going muons, as a consequence of neutrinomuon conversion inside the rock that surrounds the detector. A double differential muon flux can 


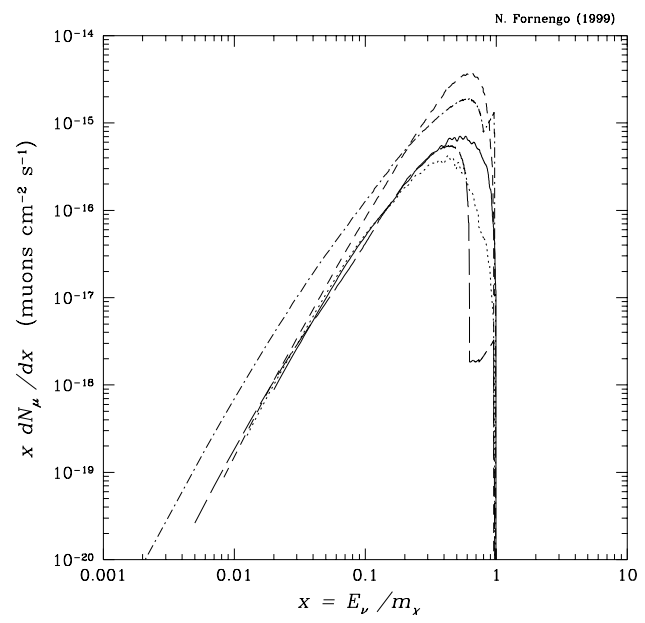

Figure 1: Muon response function $d N_{\mu} / d \log x$ vs. the parent neutrino fractional energy $x=E_{\nu} / m_{\chi}$ for neutralino annihilation in the Earth. Different curves refer to different neutralino masses : $m_{\chi}=50$ $\mathrm{GeV}$ (solid), $m_{\chi}=80 \mathrm{GeV}$ (dotted), $m_{\chi}=120 \mathrm{GeV}$ (shot-dashed), $m_{\chi}=200 \mathrm{GeV}$ (long-dash), $m_{\chi}=$ $500 \mathrm{GeV}$ (dot-dashed).

be defined as

$$
\begin{aligned}
& \frac{d^{2} N_{\mu}}{d E_{\mu} d E_{\nu}}= \\
& N_{A} \int_{0}^{\infty} d X \int_{E_{\mu}}^{E_{\nu}} d E_{\mu}^{\prime} g\left(E_{\mu}, E_{\mu}^{\prime} ; X\right) S\left(E_{\nu}, E_{\mu}\right)
\end{aligned}
$$

where $N_{A}$ is the Avogadro's number, $g\left(E_{\mu}, E_{\mu}^{\prime} ; X\right)$ is the survival probability that a muon of initial energy $E_{\mu}^{\prime}$ will have a final energy $E_{\mu}$ after propagating along a distance $X$ inside the rock and

$$
S\left(E_{\nu}, E_{\mu}\right)=\sum_{i} \Phi_{i}\left(E_{\nu}\right) \frac{d \sigma_{i}\left(E_{\nu}, E_{\mu}^{\prime}\right)}{d E_{\mu}^{\prime}}
$$

where $i=\nu_{\mu}, \bar{\nu}_{\mu}$ and $d \sigma_{(-)}\left(E_{\nu}, E_{\mu}^{\prime}\right) / d E_{\mu}^{\prime}$ is the charged current cross-section for the production of a muon of energy $E_{\mu}^{\prime}$ from a neutrino (antineutrino) of energy $E_{\nu}$.

A useful quantity for the discussion in the following Sections is the muon response function

$$
\frac{d N_{\mu}}{d E_{\nu}}=\int_{E^{\mathrm{th}}}^{E_{\nu}} d E_{\mu} \frac{d^{2} N_{\mu}}{d E_{\mu} d E_{\nu}}
$$

where $E^{\text {th }}$ is minimal energy for detection of upgoing muons. For SuperKamiokande and MACRO, $E^{\text {th }} \simeq 1.5 \mathrm{GeV}\left[\begin{array}{l}0.5 \\ 0\end{array}\right]$. The muon response function indicates the neutrino energy range that is

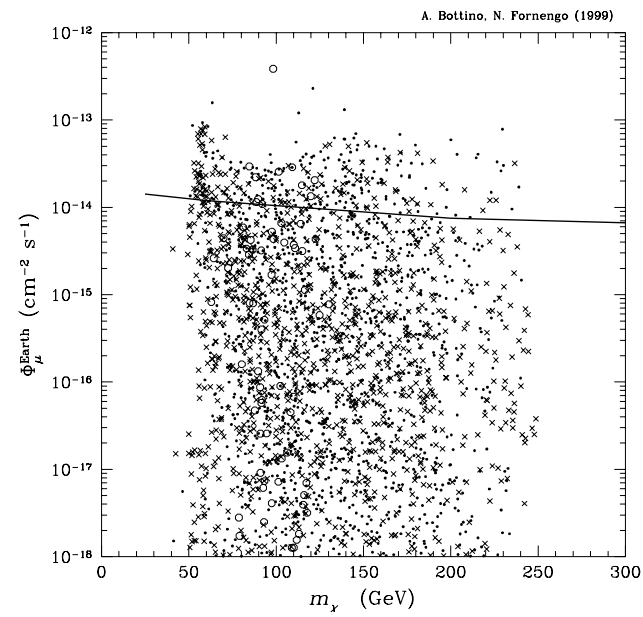

Figure 2: Flux of up-going muons $\Phi_{\mu}^{\text {Earth }}$ from neutralino annihilation in the Earth, plotted as a function of $m_{\chi}$. The solid line denotes the present upper limit [6]. Different neutralino compositions are shown with different symbols: crosses for gauginos, open circles for higgsinos and dots for mixed neutralinos.

mostly responsile for the up-going muon signal. Fig. 1 shows a few examples of it, plotted as functions of the variable $x=E_{\nu} / m_{\chi}$, where $m_{\chi}$ denotes the neutralino mass. Fig. 1 shows that the maximum of the muon reponse happens for neutrino energies of about $E_{\nu} \simeq(0.4-0.6) m_{\chi}$, with a half width which extends from $E_{\nu} \simeq 0.1 m_{\chi}$ to $E_{\nu} \simeq 0.8 m_{\chi}$.

Finally, the total flux of up-going muons is defined as

$$
\Phi_{\mu}=\int_{E^{\mathrm{th}}}^{m_{\chi}} d E_{\nu} \frac{d N_{\mu}}{d E_{\nu}}
$$

The natural background for these kind of searches is represented by the flux of up-going muons originated by the atmospheric neutrino flux. Experimentally one searches, inside a small angular cone around the center of the Earth, for a statistically significant up-going muon excess over the muons of atmospheric $\nu_{\mu}$ origin. No excess has been found so far and therefore, an upper limit on $\Phi_{\mu}$ can be derived. Fig. 2 shows the present most stringent upper limit obtained by the MACRO Collaboration [i] same figure the theoretical calculations of $\nu_{\mu}$ for a scan of the supersymmetric parameter space are also displayed. The plot refers to $\rho_{l}=0.3 \mathrm{GeV}$ 
$\mathrm{cm}^{-3}$ and is obtained by a variation of the MSSM parameters in the following ranges: $20 \mathrm{GeV} \leq$ $M_{2} \leq 500 \mathrm{GeV}, 20 \mathrm{GeV} \leq|\mu| \leq 500 \mathrm{GeV}$, $80 \mathrm{GeV} \leq m_{A} \leq 1000 \mathrm{GeV}, 100 \mathrm{GeV} \leq m_{0} \leq$ $1000 \mathrm{GeV},-3 \leq \mathrm{A} \leq+3,1 \leq \tan \beta \leq 50$. For further details of the calculation, we refer to Ref. [ind. The comparison of the scatter plot with the experimental upper limit would imply that a fraction of the supersymmetric configuration could be excluded. However, a variation of the value of $\rho_{l}$ inside its range of uncertainty can lower the theoretical prediction by about a factor of $3[2 \pi$. As a consequence, we can conservatively consider that only a small fraction of the susy configurations can be potentially in conflict with the experimental upper limit, when no oscillation effect on the neutrino signal is assumed.

\section{Neutrino oscillation effect on the up-going muon signal}

The recent data on the atmospheric neutrino deficit indicate that the $\nu_{\mu}$ may oscillate, either into

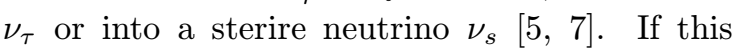
is the case, also the $\nu_{\mu}$ produced by neutralino annihilations would undergo an oscillation process. The energies involved in both atmospheric and neutralino-produced neutrinos are the same. The baseline of oscillation of the two neutrino components is different, since atmospheric neutrinos cross the entire Earth, while neutrinos produced by neutralino annihilation travel from the central part of the Earth to the detector (we recall once more that neutralinos annihilate in the core of the Earth). On the basis of the features of the $\nu_{\mu}$ oscillation which are required to fit the experimental data on atmospheric neutrinos $\left[\begin{array}{l}51 \\ 1\end{array}, \overline{7}, \overline{1}\right.$, we expect that also the neutrino flux from dark matter annihilation would be affected. In the next Sections we will explicitely discuss the $\nu_{\mu} \rightarrow \nu_{\tau}$ and the $\nu_{\mu} \rightarrow \nu_{s}$ cases, in a two neutrino mixing scenario [in].

\section{$3.1 \nu_{\mu} \rightarrow \nu_{\tau}$ vacuum oscillation}

In the case of $\nu_{\mu} \rightarrow \nu_{\tau}$ oscillation, the $\nu_{\mu}$ flux is reduced because of oscillation, but we have to take into account also that neutralino annihilation can produce $\nu_{\tau}$ which in turn can oscillate into $\nu_{\mu}$ and contribute to the up-going muon

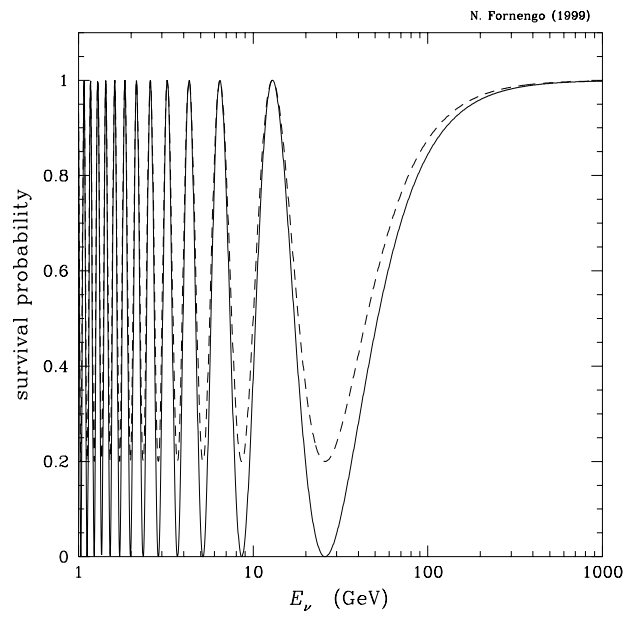

Figure 3: $\nu_{\mu}$ survival probability in the case of $\nu_{\mu} \rightarrow$ $\nu_{\tau}$ oscillation. The solid line refers to $\sin ^{2}\left(2 \theta_{v}\right)=1$, the dashed line is for $\sin ^{2}\left(2 \theta_{v}\right)=0.8$. In both cases, $\Delta m^{2}=5 \cdot 10^{-3} \mathrm{eV}^{-2}$.

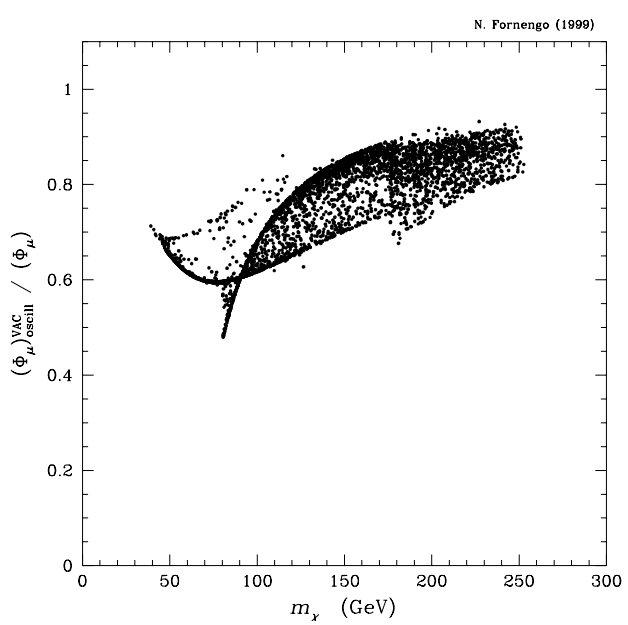

Figure 4: Scatter plot of the ratio $\left(\Phi_{\mu}\right)_{\mathrm{oscill}}^{\mathrm{VAC}} / \Phi_{\mu}$ vs. the neutralino mass $m_{\chi} .\left(\Phi_{\mu}\right)_{\mathrm{oscill}}^{\mathrm{VAC}}$ is the up-going muon flux in the case of $\nu_{\mu} \rightarrow \nu_{\tau}$ oscillation, while $\Phi_{\mu}$ is the corresponding flux in the case of no oscillation.

flux. The $\nu_{\tau}$ flux can be calculated as discussed in Sect. 1 for the $\nu_{\tau}$ flux, and it turns out to be always a relatively small fraction of the $\nu_{\mu}$ flux. The muon neutrino flux at the conversion region can therefore be expressed as

$$
\begin{aligned}
\Phi_{(-)}\left(E_{\nu}\right) & =\underset{\nu_{\mu}}{\Phi_{\nu_{\mu}}^{0}} P^{\mathrm{vac}}\left(\stackrel{(-)}{\nu_{\mu}} \rightarrow \stackrel{(-)}{\nu_{\mu}}\right) \\
& +\Phi_{\substack{(-) \\
\nu_{\tau}}}^{0} P^{\operatorname{vac}}\left(\stackrel{(-)}{\nu_{\tau}} \rightarrow \stackrel{(-)}{\nu_{\mu}}\right)
\end{aligned}
$$


where the vacuum survival probability is

$$
\begin{aligned}
& P^{\mathrm{vac}}\left(\stackrel{(-)}{\nu_{\mu}} \rightarrow \stackrel{(-)}{\nu_{\mu}}\right)= \\
& 1-\sin ^{2}\left(2 \theta_{v}\right) \sin ^{2}\left(\frac{1.27 \Delta m^{2}\left(\mathrm{eV}^{2}\right) R(\mathrm{Km})}{E_{\nu}(\mathrm{GeV})}\right)
\end{aligned}
$$

where $\Delta m^{2}$ is the mass square difference of the two neutrino mass eigenstates, $\theta_{v}$ is the mixing angle in vacuum and $R$ is the Earth's radius. Fig. 3 shows the survival probability for two different values of the neutrino oscillation parameters. Smaller (larger) values of $\Delta m^{2}$ have the effect of shifting the curves to the left (right). Comparing Fig. 1 with Fig. 3, we notice that the reduction of the up-going muon flux is stronger when there is matching between the the energy $E_{\nu}^{1} \simeq 5.2 \cdot 10^{-3} \Delta m^{2}\left(\mathrm{eV}^{2}\right)$ of the first (from the right) minimum of the survival probability and the energy $E_{\nu} \simeq 0.5 m_{\chi}$ which is responsible for most of the muon response in the detector. This implies that a maximum reduction of the signal could occur for neutralino masses of the order of $m_{\chi}(\mathrm{GeV}) \simeq 10^{4} \Delta m^{2}\left(\mathrm{eV}^{2}\right)$. The $\nu_{\tau} \rightarrow \nu_{\mu}$ oscillation makes the reduction of the muon flux less severe, but it is not able to completely balance the reduction effect because the original $\nu_{\tau}$ flux at the source is sizeably smaller than the $\nu_{\tau}$ flux. Therefore, the overall effect of the neutrino oscillation is to reduce the up-going muon signal. This effect is summarized in Fig. 4, where the ratio between the up-going muon signals in the presence and in the absence of oscillation are plotted as a function of the neutralino mass. The susy parameter space has been varied in the same ranges quoted for Fig. 2. We notice that the strongest effect is present for light neutralinos, since in this case the muon flux is mostly produced from neutrinos whose energy is in the range of maximal suppression for the oscillation phenomenon. The effect is between 0.5 and 0.8 for $m_{\chi} \lesssim 100 \mathrm{GeV}$. On the contrary, the fluxes for larger masses are less affected, and the reduction is less than about $20 \%$ for $m_{\chi} \gtrsim 200 \mathrm{GeV}$.

\section{$3.2 \nu_{\mu} \rightarrow \nu_{s}$ matter oscillation}

In the case of $\nu_{\mu} \rightarrow \nu_{s}$ oscillation, the neutrino flux is simply

$$
\Phi_{\substack{(-) \\ \nu_{\mu}}}\left(E_{\nu}\right)=\underset{\substack{\nu_{\mu} \\(-)}}{0} P^{\mathrm{mat}}\left(\stackrel{(-)}{\nu_{\mu}} \rightarrow \stackrel{(-)}{\nu_{\mu}}\right)
$$

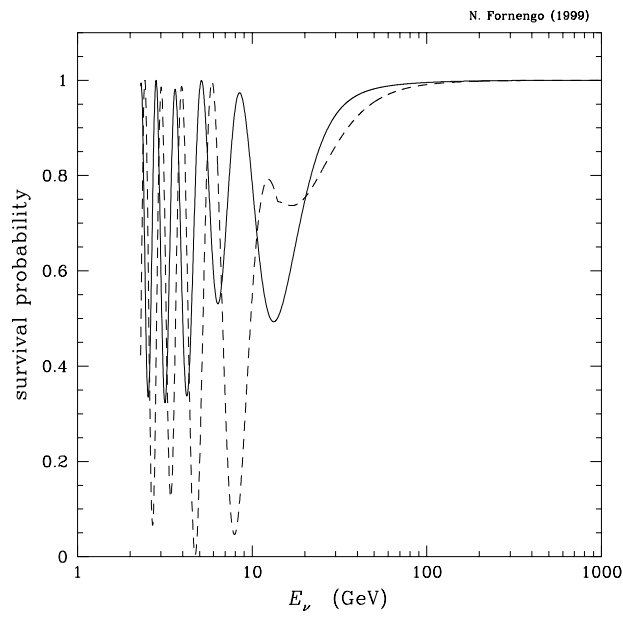

Figure 5: $\nu_{\mu}$ survival probability in the case of $\nu_{\mu} \rightarrow$ $\nu_{s}$ oscillation, for $\sin ^{2}\left(2 \theta_{v}\right)=0.8$ and $\Delta m^{2}=5 \cdot 10^{-3}$ $\mathrm{eV}^{-2}$. The solid line refers to neutrinos, the dashed line is for antineutrinos.

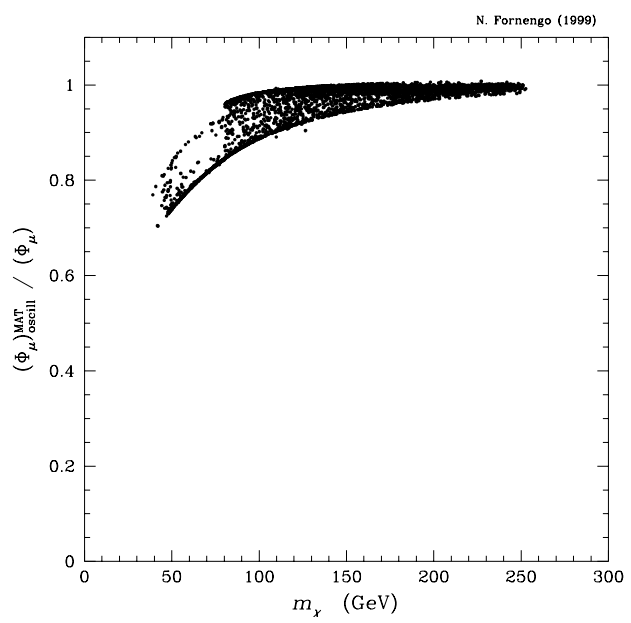

Figure 6: Scatter plot of the ratio $\left(\Phi_{\mu}\right)_{\mathrm{oscill}}^{\mathrm{MAT}} / \Phi_{\mu}$ vs. the neutralino mass $m_{\chi} . \quad\left(\Phi_{\mu}\right)_{\text {oscill }}^{\mathrm{MAT}}$ is the up-going muon flux in the case of $\nu_{\mu} \rightarrow \nu_{s}$ oscillation, while $\Phi_{\mu}$ is the corresponding flux in the case of no oscillation.

and no $\nu_{\mu}$ regeneration is possible from the sterile neutrino. In this case, the effective potential of $\nu_{\mu}$ and $\nu_{s}$ inside the Earth are different and we have to solve the evolution equation for propagation in the core and in the mantle. Neutrinos (produced in the center of the Earth) cross once half of the core and once the mantle. By considering both core and mantle as of constant density, we can express the survival probability as $[\overline{9}, 1,10$

$$
P^{\operatorname{mat}}\left(\stackrel{(-)}{\nu_{\mu}} \rightarrow \stackrel{(-)}{\nu_{\mu}}\right)=
$$




$$
\left[U\left(\theta_{c}\right) D\left(\phi_{c}\right) U^{\dagger}\left(\theta_{c}-\theta_{m}\right) D\left(\phi_{m}\right) U^{\dagger}\left(\theta_{m}\right)\right]_{\mu \mu}
$$

where $U$ is the $2 \times 2$ neutrino mixing matrix, $\theta_{a}$ ( $a=c, m$ for core and mantle, respectively) are the effective mixing angles in matter and they are related to the vacuum mixing angle $\theta_{v}$ as

$$
\sin ^{2}\left(2 \theta_{a}\right)=\frac{\sin ^{2}\left(2 \theta_{v}\right) \xi_{a}^{2}}{\left[\left(\xi_{a} \cos \left(2 \theta_{v}\right)+1\right)^{2}+\xi_{a}^{2} \sin ^{2}\left(2 \theta_{v}\right)\right]}
$$

with $\xi_{a}=\Delta m^{2} /\left(2 E_{\nu} V_{a}\right) ; \quad V_{a}= \pm G_{F} N_{n}^{a} / \sqrt{2}$ is the matter potential in a medium of number density $N_{n}^{a}$ for neutrinos (+) and antineutrinos (-). In Eq.(3.4), $D$ is the evolution matrix $D_{i j}\left(\phi_{a}\right)=\delta_{i j} d_{j}^{a}$, where $d_{1}^{a}=1, d_{2}^{a}=\exp \left(i \phi_{a}\right)$ and

$$
\phi_{a}=V_{a} R_{a}\left[\left(\xi_{a} \cos \left(2 \theta_{v}\right)+1\right)^{2}+\xi_{a}^{2} \sin ^{2}\left(2 \theta_{v}\right)\right]^{1 / 2}
$$

In Fig. 5 an example of the $\nu_{\mu}$ and $\bar{\nu}_{\mu}$ survival probability is given for representative values in the range allowed by the fits on the atmospheric neutrino data $[\overline{5}, \overline{1}, \overline{7}]: \Delta m^{2}=5 \cdot 10^{-3} \mathrm{eV}^{2}$ and $\sin ^{2}\left(2 \theta_{v}\right)=0.8$. From Fig. 5 and the previous discussion relative to Fig. 3, we expect that in the case of $\nu_{\mu} \rightarrow \nu_{s}$ the reduction of the muon signal is significantly less severe than in the case of $\nu_{\mu} \rightarrow \nu_{\tau}$. In fact, in these case the minima of the survival probability occur for lower neutrino energies, and threfore the oscillation can affect only muon fluxes originated by very light neutralinos. This is manifest in Fig. 6, where the ratio of the up-going muon fluxes in presence and absence of oscillation are shown. In - this- ease, the rectuction- of- thre- signal- is-always - fess than 30\%. - This maximal- reduction -occurs for neutralino masses lower that about $80 \mathrm{GeV}$. For larger masses, the up-going muon flux is almost unaffected.

\section{Conclusions}

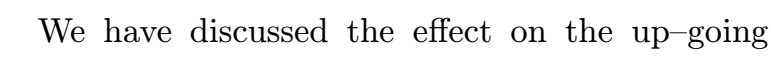
muon signal from neutratino annihitation in the Earth, in the case that the $\nu_{\mu}$ flux produced by neutralinos would oscillate as indicated by the data on the atmospherice neutrino deficit. While the experimental upper limit is, at present, practically not affected by the possibility of neutrino oscillation $[\overline{\hat{6}} \mid \overline{]}]$, the theoretical predictions are reduced in the presence of oscillation. With the oscillation parameters deduced from the fits on the atmospheric neutrino data, the effect is always larger for lighter neutralinos. In the case of $\nu_{\mu} \rightarrow \nu_{\tau}$ the reduction is between 0.5 and 0.8 for $m_{\chi} \lesssim 100 \mathrm{GeV}$ and less than about $20 \%$ for $m_{\chi} \gtrsim 200 \mathrm{GeV}$. In the case of $\nu_{\mu} \rightarrow \nu_{s}$, the reduction of the signal is up to $30 \%$ for neutralino masses lower that about $80 \mathrm{GeV}$ and smaller than $10 \%$ for heavier neutralinos.

\section{Acknowledgments}

I wish to thank Sandro Bottino for very stimulating and interesting discussions about the topic of this paper. This work was supported by DGICYT under grant number PB95-1077 and by the TMR network grant ERBFMRXCT960090 of the European Union.

\section{References}

[1] For an introduction to dark matter and a recent review on neutralino detection rates, see Ref.

[2] A. Bottino and N. Fornengo, Report. no. DFTT 20/99, FTUV/99-24, IFIC/99-26, proceedings of the Fifth School on Non-Accelerator Particle Astrophysics, Abdus Salam International Centre for Theoretical Physics, Trieste, June 1998, ed. G. Giacomelli and N. Paver (to appear).

[3] A.Bottino, N.Fornengo, G.Mignola, L.Moscoso, Astropart. Phys. 3 (1995) 65.

[4] L. Bergström, J. Edsjö and P. Gondolo, 'Ph Rev. D 58 (1998) 103519'.

[5] A. Habig (Super-Kamiokande Collaboration), hep-ex/9903047; T. Montaruli (MACRO Collaboration), hep-ex/9810017.

[6] The MACRO Collaboration, M. Ambrosio et al., hep-ex/9812020!

[7] M.C. Gonzalez-Garcia, H. Nunokawa, O.L.G. Peres, J.W.F. Valle, 'Nucl. Phys. B $\mathbf{5 4 3}(1999)$ 3.

[8] The possibility of neutrino oscillation on the indirect signal from neutralino annihilation has also been considered in J. Ellis, R.A. Flores and S.S. Masood, Phys. Lett. B 294 (1992) 2299 Phys. Lett. B294, $229(1992)$ in the case of the signal from the Sun. 
[9] E. Akhmedov, 1Yad. Fiz. 47 (1988) 475! [Sov. J. Nucl. Phys. 47 (1988) 301].

[10] C.W. Kim and A. Pevsner, Neutrinos in Physics and Astrophysics, Contemporary Concepts in Physics, vol. 8 (Harwood Academic Press, Chur, switzerland, 1993). 